\title{
Spatial structure and dispersion of drift mirror waves coupled with Alfvén waves in a 1-D inhomogeneous plasma
}

\author{
D. Yu. Klimushkin \\ Institute of Solar-Terrestrial Physics (ISTP), Russian Academy of Science, Siberian Branch, Irkutsk, 664033, Russia
}

Received: 1 March 2006 - Accepted: 17 July 2006 - Published: 13 September 2006

\begin{abstract}
The paper employs the frame of a 1-D inhomogeneous model of space plasma,to examine the spatial structure and growth rate of drift mirror modes, often suggested for interpreting some oscillation types in space plasma. Owing to its coupling with the Alfvén mode, the drift mirror mode attains dispersion across magnetic shells (dependence of the frequency on the wave-vector's radial component, $k_{r}$ ). The spatial structure of a mode confined across magnetic shells is studied. The scale of spatial localization of the wave is shown to be determined by the plasma inhomogeneity scale and by the azimuthal component of the wave vector. The wave propagates across magnetic shells, its amplitude modulated along the radial coordinate by the Gauss function. Coupling with the Alfvén mode strongly influences the growth rate of the drift mirror instability. The mirror mode can only exist in a narrow range of parameters. In the general case, the mode represents an Alfvén wave modified by plasma inhomogeneity.
\end{abstract}

Keywords. Magnetospheric physics (Magnetosheath; MHD waves and instabilities) - Space plasma physics (Kinetic and MHD theory)

\section{Introduction}

Compressional storm-time Pc5 pulsation within the magnetosphere (Takahashi, 1996; Woch et al., 1990) and some typs of waves observed in the magnetosheath (Denton, 2000; Lucek et al., 2005; Narita and Glassmeier, 2005) have in the past been identified with the drift-mirror mode. This mode, being compressional (i.e. exhibiting a significant variation of the absolute value of the magnetic field which, in the linear approximation, means the presence of a longitudinal component in the wave magnetic field), is akin to the fast

Correspondence to: D. Klimushkin

(klimush@iszf.irk.ru) magnetic sound (FMS). A generalization of the FMS dispersion equation for the case of finite-pressure plasma oscillations, obtained by solving the Vlasov equation, has the form (Hasegawa, 1975)

$$
\begin{aligned}
& \left(\frac{\omega^{2}}{v_{A}^{2}}-k_{\|}^{2}-k_{\perp}^{2}\right)+k_{\|}^{2} \frac{V_{\|}^{2}-V_{\perp}^{2}}{2 v_{A}^{2}} \\
& \quad-\beta_{\perp} k_{\perp}^{2}\left\{1-\frac{T_{\perp}}{T_{\|}}\left[1+Z\left(\frac{\omega}{\sqrt{2} k_{\|} V_{\|}}\right)\right]\right\}=0 .
\end{aligned}
$$

Here $\omega$ is the wave frequency, $v_{A}$ is the Alfvén velocity, $k_{\|, \perp}$ are the wave vector components along and across the equilibrium magnetic field, $V_{\|, \perp}$ and $T_{\|, \perp}$ are the thermal velocities of particles and temperatures along and across the field, $\beta_{\|, \perp}$ is the ratio between the longitudinal or transverse pressure of the plasma and magnetic pressure, $Z$ is the FriedConte plasma dispersion function. Under vanishingly small pressure, the usual FMS dispersion equation follows from Eq. (1): $\omega^{2}=\left(k_{\|}^{2}+k_{\perp}^{2}\right) v_{A}^{2}$. The mirror mode represents another limit, when the plasma and magnetic pressure are of the same order of magnitude, $\beta \sim 1$, with the transverse wave vector being large, $k_{\perp} \gg k_{\|}, \omega / v_{A}$, and the frequency very low, $\omega \ll k_{\|} V_{\|}$. In this case, as implied by Eq. (1), the frequency is purely imaginary: $\omega=i \gamma_{M}$, where

$\gamma_{M}=-\tau \sqrt{\frac{2}{\pi}} \frac{k_{\|} V_{\|}}{\beta_{\perp}} \frac{T_{\|}}{T_{\perp}}$

and

$\tau=1+\beta_{\perp}\left(1-\frac{T_{\perp}}{T_{\|}}\right)$.

When $\tau<0$, the disturbance increases (mirror instability). For a more detailed discussion of the physical nature of this mode see Hasegawa (1969); Southwood and Kivelson (1993); Treumann (2001); Treumann et al. (2004). In an in-

Published by Copernicus GmbH on behalf of the European Geosciences Union. 
homogeneous plasma, the mirror mode frequency acquires a real part

$\omega_{*}=\frac{k_{y} V_{\|}^{2}}{\omega_{c}}\left[(\log n)^{\prime}+3 \alpha\left(\log B_{0}\right)^{\prime}\right]$

$\left(\omega_{c}\right.$ is a gyrofrequency, $n$ is a concentration, $\left.\alpha \equiv\left(T_{\|}-T_{\perp}\right) / T_{\perp}\right)$, whose order of magnitude coincides with the drift frequency (Hasegawa, 1969; Pokhotelov et al., 2001). The parameter $\alpha$ characterizes the plasma anisotropy. In ordinary theory the parameter $\alpha$ determines the nature of the instability: $\alpha>0$ is required for the firehose instability of Alfvén waves, while $\alpha<0$ holds for the mirror instability. Hence, in ordinary theory both instabilities behave complimentary. But the drift mirror modes are coupled with transverse Alfvén waves in an inhomogeneous plasma (Lin and Parks, 1978; Chen and Hasegawa, 1991; Ferrière and Andre, 2003, 2005).

A number of further questions arise when studying drift mirror waves in inhomogeneous plasma. First of all, the dispersion relation (2) does not contain the transverse component $k_{\perp}$ of the wave vector, i.e. these waves lack transverse dispersion. Transverse dispersion does appear when the finite ion Larmor radius effects are taken into account (Pokhotelov et al., 2004), but we are dealing with much longer waves, in which these effects are weak. The same is true for Alfvén waves, but they see transverse dispersion when curved field lines and finite plasma pressure are accounted for (Leonovich and Mazur, 1993; Klimushkin, 1997; Mager and Klimushkin, 2002). Is it not the case for the drift mirror mode as well?

This question is closely related to what spatial structure these waves may have in inhomogeneous plasma. The Alfvén mode with a given $\omega$ and $k_{\|}$is known to be narrowly localized close to a magnetic shell near where the relationship $\omega=k_{\|} v_{A}(r)$ (here $r$ is the coordinate across magnetic shells) holds. An analogous expression for the drift mirror waves is $\operatorname{Re} \omega=\omega_{*}(r)$. Does it not imply that the drift mirror mode is also narrowly localized close to a certain magnetic surface? Further, some papers (e.g. Hasegawa, 1969; Lin and Parks, 1978 and others) examined drift mirror modes with $k_{r} \ll k_{y}$, where $k_{r}$ and $k_{y}$ are the wave vector components across magnetic shells and along the binormal to field lines, respectively. Under what conditions is this possible? In particular, the inequality $k_{r} \ll k_{y}$ naturally holds for modes confined across magnetic shells between two reflection points on the radial coordinate $r$. Can such modes exist?

The studies of mode stability and spatial structure are closely interrelated. Thus, in the case of a mode trapped across magnetic shells, its structure is determined by solving a certain differential equation whose eigen value is frequency, including its imaginary part, the instability growth rate. For the Alfvén waves, this procedure was done in Vetoulis and Chen (1996). It would be interesting to perform the same for the drift mirror mode.
The next question relates to the ratio $\omega \ll k_{\|} V_{\|}$(the "mirror limit"). As follows from Eq. (2), this inequality can only be satisfied for $\tau \ll 1$. But the quantity $\tau$ is a combination of order-unity terms, and there are no specific reasons for its absolute value to be much smaller than unity, including under strong plasma anisotropy. Which modes with $\omega \ll k_{\|} V_{\|}$exist in inhomogeneous plasma with $\tau \sim 1$ ?

Finding answers to these questions is the aim of this paper.

\section{The model and major equations}

The paper considers a model magnetosphere with equilibrium magnetic field $B_{0}$ lines with constant curvature. All the plasma parameters vary across magnetic shells only. The plasma to magnetic pressure ratio is $\beta \sim 1$, but the plasma has a population of cold electrons, providing a shorted-out longitudinal electric field $\left(E_{\|}=0\right)$. The same-sign particle concentration is $n$. The equilibrium distribution function $F$ is assumed to be bi-Maxwellian, the mean motion velocities along and across the field are $V_{\|}$and $V_{\perp}$. The longitudinal and transverse components of the equilibrium pressure are $P_{\|}$and $P_{\perp}$. Plasma temperature is assumed to be unchanging in the entire volume. Plasma anisotropy is characterized by the parameter $\alpha=\left(T_{\|}-T_{\perp}\right) / T_{\perp}$.

For $E_{\|}=0$ the wave can be described by two variables: $\Psi$, related to the longitudinal vector potential $A_{\|}$by $A_{\|}=-(i c / \omega) \partial \Psi / \partial l_{\|}$, where $\omega$ is the wave frequency; and $b=\omega B_{\|} / c$, where $B_{\|}$is the longitudinal magnetic field (Chen and Hasegawa, 1991). The dependence of the wave variables on the spatio-temporal coordinates has the form

$\Psi, b \propto \exp \left[-i \omega t+i k_{y} y+i \int k_{r}(r) d r+i k_{\|} l_{\|}\right]$,

where $r$ is the radial coordinate (field-line curvature radius), $y$ is the azimuthal coordinate, $l_{\|}$is the longitudinal coordinate (along the two latter, the equilibrium parameters are assumed unchanged), $k_{r}$ is the wave-vector's radial component determined from the leading order of the WKB approximation.

The field variables are related by the gyrokinetic equations (Catto et al., 1981; Antonsen and Lane, 1980) which are conveniently re-written as (Chen and Hasegawa, 1991)

$L_{M} b-k_{y} L_{c} \Psi=0$,

$L_{A} \Psi-k_{y} L_{c} b=0$.

The following notation is introduced here:

$L_{M}=\tau+a_{M}$,

where

$\tau=1+\frac{8 \pi^{2}}{c^{2}} \sum_{e, i} \frac{q^{2}}{m} \int d v_{\|} d v_{\perp} v_{\perp}^{3} J_{1}^{2}(\xi)\left(\frac{F}{V_{\perp}^{2}}-\frac{F}{V_{\|}^{2}}\right)$, 
$a_{M}=\frac{4 \pi \omega^{2}}{c^{2}} \frac{1}{k_{\|} k_{\perp}^{2}} 2 \pi \sum_{e, i} \frac{q^{2}}{m} \int d v_{\|} d v_{\perp} v_{\perp}^{3} J_{1}^{2}(\xi) \hat{Q} F$,

$\varepsilon=v^{2} / 2$ is the particle energy, $\xi=k_{\perp} v_{\perp} / \omega_{c}$ is an argument of the Bessel function,

$\hat{Q} F=\left(v_{\|}-\frac{\omega-\omega_{d}}{k_{\|}}\right)^{-1}\left(\frac{\partial F}{\partial \varepsilon}+\frac{k_{y} F^{\prime}}{\omega \omega_{c}}\right)$,

and

$\omega_{d}=\frac{k_{y}}{\omega_{c}}\left(\frac{B_{0}^{\prime}}{2 B_{0}} v_{\perp}^{2}-\frac{v_{\|}^{2}}{r}\right)$

is the drift frequency in inhomogeneous magnetic field (prime means differentiated over radius $r$ ). Further,

$$
\begin{aligned}
L_{A} & =\left(k_{r}^{2}+k_{y}^{2}\right)\left(\sigma k_{\|}^{2}-\frac{\omega^{2}}{v_{A}^{2}}\right) \\
& -k_{y}^{2} \frac{4 \pi}{B_{0}^{2}}\left(P_{\perp}^{\prime} \frac{B_{0}^{\prime}}{B_{0}}-\frac{P_{\|}^{\prime}}{r}\right)-a_{A},
\end{aligned}
$$

where $\sigma=1-(1 / 2)\left(\beta_{\|}-\beta_{\perp}\right)$ and

$a_{A}=\frac{4 \pi \omega^{2}}{c^{2}} \frac{\omega}{k_{\|}} 2 \pi \sum_{e, i} \frac{q^{2}}{m} \int d v_{\|} d v_{\perp} v_{\perp}\left(\frac{\omega_{d}}{\omega}\right)^{2} J_{0}^{2}(\xi) \hat{Q} F$.

Finally,

$L_{c}=\frac{4 \pi P_{\perp}^{\prime}}{B_{0}^{2}}-a_{c}$,

$a_{c}=\frac{4 \pi \omega^{2}}{c^{2}} \frac{2 \pi}{k_{\|} k_{\perp}} \sum_{e, i} \frac{q^{2}}{m} \int d v_{\|} d v_{\perp} v_{\perp}^{2} J_{0}(\xi) J_{1}(\xi) \frac{\omega_{d}}{\omega} \hat{Q} F$.

Note that the terms $a_{M}, a_{A}, a_{c}$ are responsible for the waveparticle interaction.

Note that the operators $L_{M}$ and $L_{A}$ are often called, respectively, the compressional (mirror) and transverse (Alfvén) mode operators. The operator $L_{c}$ is then responsible for coupling of these modes. Such terminology originates from the theory of weakly coupled oscillation modes in homogeneous plasma. In the inhomogeneous plasma case the system $(5,6)$ should be understood somewhat differently: as the leading order of the WKB approximation of a differential equation system describing the wave's transverse $(\Psi)$ and longitudinal $(b)$ magnetic field. Solving this problem we find the functions $\Psi(r)$ and $b(r)$ containing information on the mode localization areas. Thus, from the system (5, 6) we eventually determine where in the plasma the wave is localized and what polarization it has in these regions. In inhomogeneous plasma, there is no need whatsoever to single out the Alfvén and compressional components from the total wave field as being somewhat different from each other.
Expressing $b$ from Eq. (5) and substituting it into Eq. (6), we obtain

$L_{A}-k_{y}^{2} \frac{L_{c}^{2}}{L_{M}}=0$.

This expression should be considered as an equation relating the radial component $k_{r}(r)$ of the wave vector to frequency $\omega$, corresponding to the leading order of the WKB approximation.

In this paper we will neglect the particle Larmor radius $(\xi \ll 1)$ and the pressure of the plasma electron component. Then, in the mirror limit $\omega, \omega_{d} \ll k_{\|} V_{\|}$, the operators are recast as

$$
\begin{aligned}
L_{M} & =\tau-i \delta\left(1-\frac{\omega_{*}}{\omega}\right), \\
L_{A} & =k_{\perp}^{2}\left(\sigma k_{\|}^{2}-\frac{\omega^{2}}{v_{A}^{2}}\right)-k_{y}^{2} \frac{4 \pi}{B_{0}^{2}}\left(P_{\perp}^{\prime} \frac{B_{0}^{\prime}}{B_{0}}-\frac{P_{\|}^{\prime}}{r}\right) \\
& +i k_{y}^{2} \delta \frac{\omega-\omega_{*}}{\omega}\left(\frac{B^{\prime}}{B}\right)^{2}, \\
L_{c} & =\frac{4 \pi P_{\perp}^{\prime}}{B_{0}^{2}}+i \delta \frac{\omega-\omega_{*}}{\omega} \frac{B^{\prime}}{B} .
\end{aligned}
$$

Here is designated

$\delta=\left(\frac{\pi}{2}\right)^{1 / 2} \frac{T_{\perp}}{T_{\|}} \frac{\omega}{k_{\|} V_{\|}} \frac{8 \pi n_{h} m_{i} V_{\perp}^{2}}{B_{0}^{2}}$,

the quantities $\tau$ and $\omega_{*}$ are determined by the expressions (3) and (4).

\section{Combined study of the spatial structure and growth rate of the drift mirror mode}

If we set $L_{c}=0$, it follows from Eq. (7) that $L_{M}=0$, whence we obtain a "classical" dispersion equation for the drift mirror mode $\omega=\omega_{*}+i \gamma_{M}$, where $\gamma_{M}$ is determined by the expression (2) in the Introduction. In the general case, however, the coupling operator must not be neglected. Equation (7) is then recast as

$k_{r}^{2}(\omega, r)=-K^{2} \frac{\omega-\omega_{*}(r)-i \gamma_{M}(r)-i \Omega_{c}(r)}{\omega-\omega_{*}(r)-i \gamma_{M}(r)}$.

The following notations are introduced here:

$$
\begin{aligned}
& \Omega_{c}=\sqrt{\frac{2}{\pi}} \frac{k_{\|} V_{\|}}{\beta_{\perp}} \frac{T_{\|}}{T_{\perp}} \frac{1}{\tilde{L}_{P}}\left(\frac{4 \pi P_{\perp}^{\prime}}{B_{0}^{2}}\right)^{2}, \\
& L_{T}(\omega, r)=\sigma k_{\|}^{2}-\frac{\omega^{2}}{v_{A}^{2}}, \\
& \tilde{L}_{P}=L_{T}-\frac{4 \pi}{B_{0}^{2}}\left(P_{\perp}^{\prime} \frac{B_{0}^{\prime}}{B_{0}}-\frac{P_{\|}^{\prime}}{r}\right),
\end{aligned}
$$


$K^{2}=k_{y}^{2} \frac{\tilde{L}_{P}}{L_{T}}$.

Note that $K \sim k_{y}$. We will consider waves with $\operatorname{Re} \omega \sim \omega_{*}$. Let us introduce the quantities $\Omega_{T}$ and $\tilde{\Omega}_{P}$ determined from equations $L_{T}\left(\Omega_{T}\right)=0$ and $\tilde{L}_{P}\left(\tilde{\Omega}_{P}\right)=0$. These are certain characteristic frequencies of the Alfvén wave. Let the wave frequency $\omega$ be much less than any of them. The Alfvén operator then differs significantly from zero in the mode localization region. In this case expression (12) can be interpreted as a dispersion expression for the drift mirror mode modified by its coupling to the Alfvén mode. We can see that this modification reduces to the emergence of transverse dispersion in the drift mirror mode.

It is interesting that we come across an analogous situation when studying the slow magnetic sound (SMS) in the MHD frame. In the limit $k_{\perp} \gg k_{\|}$the SMS dispersion equation has the form $\omega=v_{S} k_{\|}\left[1+O\left(k_{\|}^{2} / k_{\perp}^{2}\right)\right]$ (where $v_{S}$ is the slow magnetic sound velocity), meaning that the SMS dispersion becomes negligibly small. But when field line curvature is taken into account, the SMS mode becomes coupled to the Alfvén mode (Klimushkin, 1997) (analogously to our case of coupled mirror-Alfvén modes). This results in recovered transverse SMS dispersion.

Equation (12) is also the leading-order equation in the WKB approximation for the drift mirror mode. This will allow us to obtain some information about the spatial structure of the drift mirror mode.

Let us consider modes with $k_{r} \simeq 0$ localized across magnetic shells. This is possible in the regions where the functions $\Omega_{c}(r)$ and $\omega_{*}(r)$ reach their extremes, their locations practically exactly coinciding. Additionally, these extrema should be in the region where $|\tau| \ll 1$. Such coincidences are rather unlikely, of course. Nevertheless, let us consider the mode structure in such a resonator.

It can be seen from Eq. (12) that for $k_{r} \simeq 0$ the real and imaginary parts of the frequency are determined by the expressions

$\operatorname{Re} \omega \simeq \omega_{* 0}, \quad \operatorname{Im} \omega \simeq \Omega_{c 0}+\gamma_{M 0}$.

The index " 0 " here denotes the extremes of corresponding quantities. For definiteness, let us consider the point $r_{0}$ where the quantities $\Omega$ and $\omega_{*}$ are positive. If their variation scales along the radial coordinate are approximately the same, we may make use of the quadratic expansions

$\Omega_{c}=\Omega_{0}\left(1-\frac{x^{2}}{l^{2}}\right)$,

$\omega_{*}=\omega_{* 0}\left(1-\frac{x^{2}}{l^{2}}\right)$.

Here $x=r-r_{0}$, and $l$ is the typical scale of plasma inhomogeneity. All the other quantities included in Eq. (12) may be considered approximately constant in the mode localization region. If the approximate Eq. (16) hold, the expression (12) reduces to

$$
\begin{aligned}
k_{r}^{2}=\frac{K^{2}}{\Omega_{0}}\left[\left(\gamma_{M 0}\right.\right. & \left.+\Omega_{0}\right)+i\left(\omega-\omega_{* 0}\right) \\
& \left.-\left(\Omega_{0}-i \omega_{* 0}\right) \frac{x^{2}}{l^{2}}\right] .
\end{aligned}
$$

The transverse small-scale condition for the mode $k_{\perp} \gg k_{\|}$ reduces ultimately to $K l \gg 1$.

Let us consider a differential equation, which in the leading order of the WKB approximation reduces to the form of Eq. (17). The simplest equation of this kind has the form

$$
\begin{aligned}
& \frac{d^{2} f}{d x^{2}}+\frac{K^{2}}{\Omega_{0}} {\left[\left(\gamma_{M 0}+\Omega_{c 0}\right)+i\left(\omega-\omega_{* 0}\right)\right.} \\
&\left.-\left(\Omega_{c 0}-i \omega_{* 0}\right) \frac{x^{2}}{l^{2}}\right] f=0 .
\end{aligned}
$$

Of course, many such equations can be introduced, differing from each other by the first derivative of the function $f(x)$ describing the wave amplitude. But the terms of the $d f / d x$ type do not influence the eigen values $\omega$, so it is sufficient to restrict ourselves to Eq. (18). The boundary condition to this equation is the boundedness of the function $f$ at $x \rightarrow \pm \infty$.

Let us introduce a new variable $\zeta=x / \lambda$, where $\lambda$ is found from the condition

$K^{2} l^{-2} \lambda^{4}\left(1-i \omega_{* 0} \Omega_{c 0}^{-1}\right)=1$.

Extracting the root from $\lambda^{4}$ we should keep in mind the condition that the function $f$ decrease when $x \rightarrow \pm \infty$. Hence, $\operatorname{Re} \lambda^{-2}>0$, i.e.

$\lambda^{2}=\frac{l}{|K|}\left(1+\frac{\omega_{* 0}^{2}}{\Omega_{c 0}^{2}}\right)^{-1 / 4} e^{i \phi / 2}$,

where

$$
\begin{aligned}
& \cos \phi=\Omega_{c 0} / \sqrt{\Omega_{c 0}^{2}+\omega_{* 0}^{2}}, \\
& \sin \phi=\omega_{* 0} / \sqrt{\Omega_{c 0}^{2}+\omega_{* 0}^{2}} .
\end{aligned}
$$

On the order of magnitude, $\lambda \sim \sqrt{l / K}$. Let us introduce the notation

$\theta=\frac{\Omega_{c 0}+\gamma_{M 0}-\gamma}{\sqrt{\left(\Omega_{c 0}+\gamma_{M 0}-\gamma\right)^{2}+\left(\omega-\omega_{* 0}\right)^{2}}}$,

where $\gamma$ is the imaginary part of the wave frequency.

Now Eq. (18) reduces to

$\frac{d^{2} f}{d \zeta^{2}}+\left(\theta-\zeta^{2}\right) f=0$.

This is the problem on the eigen values of $\theta$,

$\operatorname{Re} \theta=2 n+1, \quad \operatorname{Im} \theta=0$, 
where $n$ is an integer. From here we obtain the eigen values for the instability growth rate $\gamma$ and for the real part of the wave frequency:

$$
\begin{gathered}
\gamma_{n}=\left(\Omega_{c 0}+\gamma_{M 0}\right) \\
-\frac{2 n+1}{\sqrt{2}} \frac{\Omega_{c 0}^{1 / 2}}{k_{y} l}\left[\sqrt{\Omega_{c 0}^{2}+\omega_{* 0}^{2}}+\Omega_{c 0}\right]^{1 / 2}, \\
\operatorname{Re} \omega_{n}=\omega_{* 0}+\frac{2 n+1}{\sqrt{2}} \frac{\Omega_{c 0}^{1 / 2}}{k_{y} l}\left[\sqrt{\Omega_{c 0}^{2}+\omega_{* 0}^{2}}-\Omega_{c 0}\right]^{1 / 2}
\end{gathered}
$$

(note the correspondence of the obtained expressions to the formulas (16) that takes place for $K l \gg 1$ ). The solution of Eq. (18) is written as

$$
f(r)=\mathrm{H}_{n}\left(\frac{r-r_{0}}{\lambda}\right) \exp \left[-\frac{\left(r-r_{0}\right)^{2}}{2 \lambda^{2}}\right],
$$

where $\mathrm{H}_{n}$ is the Hermitian polynomial.

Let us discuss the solution obtained. An instability develops for $\gamma_{n}>0$. As can be seen from Eq. (21) coupling to the Alfvén mode (depicted by $\Omega_{c}$ ) substantially affects the instability. With high harmonic numbers $n \gg 1$ coupling favours plasma stability. With low numbers $n \sim 1$ the second term in Eq. (21) is considerably smaller than the first due to the large value of $K l$, i.e. coupling favours instability. A negative value of $\tau$ (included into the definition of $\gamma_{M}$ ) also encourages instability, but even with $\tau>0$ the instability is possible.

The real part of the eigenfrequency grows with increasing harmonic number. This growth is, however, rather slow due to the factor $(K l)^{-1}$ present in the second term of Eq. (22). Therefore, the wave frequency almost coincides with $\omega_{* 0}$. This is close to the ULF wave frequencies observed in the real magnetosphere (including the compressional storm-time pulsations Pc5).

Radial structure of the wave described by formula (23) is shown schematically in Fig. 1. The mode is trapped between two cutoff magnetic surfaces (see also Treumann and Baumjohann, 2000). In the general case $\Omega_{c 0} \sim \omega_{* 0}$, and the structure has an oscillatory character even for $n=0$ (Fig. 1a), with the amplitude modulated by the Gauss function. The presence of the imaginary part in $\lambda^{2}$ results in the wave propagating across magnetic shells. The real part of the wave vector's radial component is $\operatorname{Re} k_{r}=\left(r-r_{0}\right)|K| l^{-1}\left|\lambda^{-2}\right| \sin (\phi / 2)$. Since by the definition of $\phi$ (20) the sign of $\sin \phi$ coincides with that of $\omega_{* 0}$, and the quantity is positive at the point $r_{0}$, then $\sin (\phi / 2)>0$. Therefore, the wave phase velocity is directed away from the surface $r_{0}$. As can be seen from Eq. (19), meanwhile, $k_{r}$ does not depend on $\omega$, i.e. there is no energy transfer across magnetic shells (as is the case for the Alfvén wave in homogeneous plasma). The wave is standing across field lines (Fig. 1b) in the case $\sin (\phi / 2)=0$ only. As can be seen from Eq. (20) it is possible if $\omega_{* 0} \ll \Omega_{c 0}$.

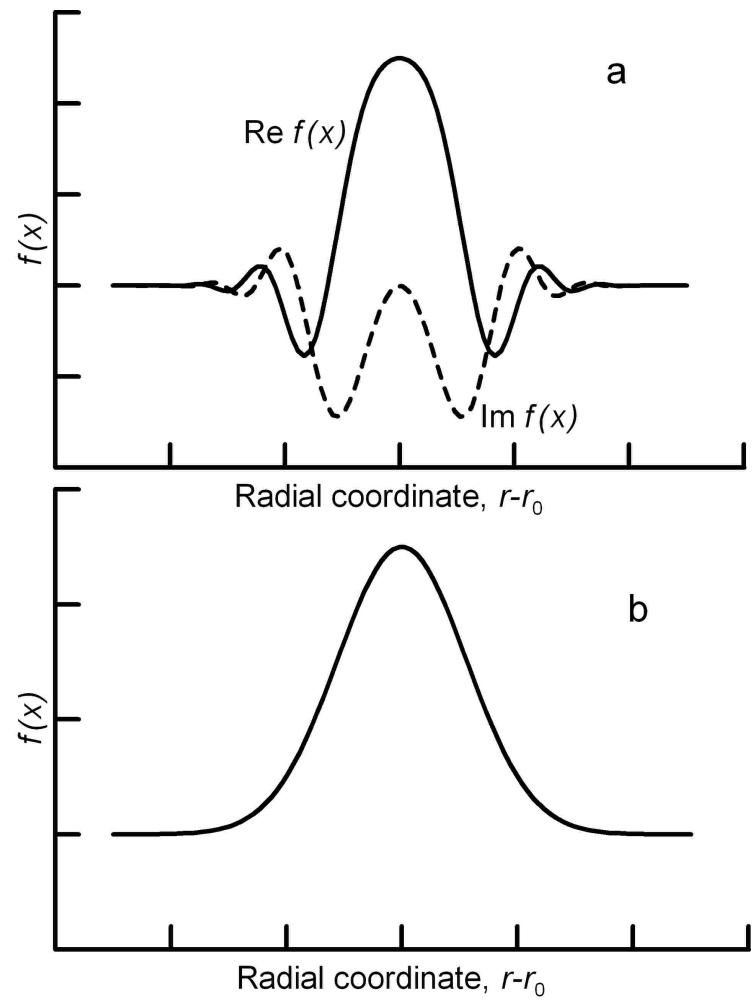

Fig. 1. Transverse structure of the mode: (a) in the $\Omega_{0} \sim \omega_{* 0}$ case (values $\operatorname{Re} f(x), \operatorname{Im} f(x)$ are shown); (b) in the $\Omega_{0} \gg \omega_{* 0}$ case.

Let us consider the fulfilment conditions for expressions obtained in this chapter. The condition $\omega \ll k_{\|} V_{\|}$places restrictions to the values of the real and imaginary parts of the wave frequency. The real wave frequency is close to the drift frequency, which on the order of magnitude is equal to $\omega_{*} \sim k_{y} \rho_{i} v_{T} l^{-1}$, where $\rho_{i}$ is the ion Larmor radius, $v_{T}$ is the typical particle thermal velocity. The inequality $\omega_{*} \ll k_{\|} V_{\|}$ is satisfied if $\left(k_{y} \rho_{i}\right) /\left(k_{\|} l\right) \ll 1$. Contribution to the imaginary wave frequency is made by $\gamma_{M}$ and $\Omega_{c}$. The condition $\gamma_{M} \ll k_{\|} V_{\|}$for $\beta_{\perp} \sim 1$ means $|\tau| \ll 1$. Finally, $\Omega_{c} \ll k_{\|} V_{\|}$is only possible if the condition $\left(k_{\|} l\right)^{2} \gg 1$ is met. The real and the imaginary parts of the frequency, in the meantime, are of the same order of magnitude.

Finally, let us consider mode polarization in the resonator. It follows from the general gyrokinetic formulas (Antonsen and Lane, 1980) that the vector potential's longitudinal component $A_{\|}$, i.e. the variable $\Psi \equiv\left(\omega / c k_{\|}\right) A_{\|}$is responsible for the wave's transverse magnetic field. Since $k_{r} \ll k_{y}$ in the resonator, we conclude that the wave's transverse magnetic field is dominated by the radial component (the wave has poloidal polarization). According to the system $(5,6)$, the transverse and longitudinal components of the magnetic field are related by

$$
\frac{B_{\perp}}{B_{\|}} \sim \frac{4 \pi P_{\perp}^{\prime}}{\sigma k_{\|} B_{0}^{2}} .
$$


In view of the condition $\left(k_{\|} l\right)^{2} \gg 1$, it follows from here that the longitudinal component of the magnetic field dominates over its transverse component.

\section{Case $\tau \sim 1$ : ballooning-compressional Alfvén mode}

As has already been noted, the quantity $\tau$ is a combination of terms of order unity and there are no special reasons for its absolute value to be much smaller than unity. The smallness of $\delta$ is, meanwhile, provided by the inequality $\omega \ll k_{\|} V_{\|}$and we can write in Eq. (7)

$\frac{1}{L_{M}}=\frac{1}{\tau}+i \frac{\delta}{\tau^{2}}\left(1-\frac{\omega_{*}}{\omega}\right)$.

Then Eq. (7) reduces to

$$
\begin{aligned}
k_{r}^{2} L_{T}(\omega, r) & +k_{y}^{2} L_{P}(\omega, r) \\
& -i k_{y}^{2} \delta \frac{\omega-\omega_{*}}{\omega}\left[\frac{B^{\prime}}{B}-\frac{4 \pi P_{\perp}^{\prime}}{\tau B_{0}^{2}}\right]^{2}=0 .
\end{aligned}
$$

Here the toroidal operator $L_{T}$ is determined by the expression (14), and we will call the operator

$$
\begin{aligned}
L_{P}(\omega, r) & =\left(\sigma k_{\|}^{2}-\frac{\omega^{2}}{v_{A}^{2}}\right)-\frac{4 \pi}{B_{0}^{2}}\left(P_{\perp}^{\prime} \frac{B_{0}^{\prime}}{B_{0}}-\frac{P_{\|}^{\prime}}{r}\right) \\
& -\frac{1}{\tau}\left(\frac{4 \pi P_{\perp}^{\prime}}{B_{0}^{2}}\right)^{2}
\end{aligned}
$$

poloidal. The additional terms in this expression, not contained in the toroidal operator expression, are called the ballooning terms.

In the homogeneous plasma limit, Eq. (25) reduces to the dispersion equation of the Alfvén mode $\omega^{2}=\sigma k_{\|}^{2} v_{A}^{2}$. Thus, Eq. (25) is the dispersion equation of a very-low-frequency $\left(\omega \ll k_{\|} V_{\|}\right)$Alfvén wave in inhomogeneous finite-pressure plasma. We may call this mode a ballooning-compressional Alfvén mode.

Let us introduce the toroidal $\Omega_{T}$ and the poloidal $\Omega_{P}$ frequency determined from the conditions

$L_{T}\left(\Omega_{T}\right)=0$

and

$L_{P}\left(\Omega_{P}\right)=0$.

In the case $\omega \simeq \Omega_{T}$, Eq. (25) implies that $k_{r} \gg k_{y}$. In this case the azimuthal component dominates in the wave magnetic field (oscillations are toroidal). Conversely, in the case $\omega \simeq \Omega_{P}$ the inequality $k_{r} \ll k_{y}$ holds, i.e. the radial component of magnetic field exceeds its azimuthal component and the oscillations are poloidal. Thus, the presence of ballooning terms leads to the polarization splitting of the spectrum. Further, the presence of ballooning terms results in the emergence of transverse dispersion in the Alfvén wave, i.e. the dependence of the frequency on the wave vector's transverse component, which is also explicit in Eq. (25). The consequence is the appearance of transverse components $v_{g \perp}=\partial \omega / \partial k_{\perp}$ in the group velocity.

Imaginary terms in Eq. (25) are responsible for collisionless damping or swinging of the Alfvén wave. Notice that the inequality $\omega \ll k_{\|} V_{\|}$determines the smallness of the imaginary part in the frequency compared to its real part, in contrast to the drift mirror mode.

\section{Conclusions}

Here are the chief conclusions of the paper.

1. In the general case, when parameter $|\tau| \sim 1$, even if it is negative, waves with $\omega \ll k_{\|} V_{\|}$are Alfvén waves modified by the plasma inhomogeneity and field-line curvature. This modification consists of the appearance in the dispersion equation of ballooning terms leading to differing frequencies in poloidal and toroidal oscillations, the appearance of transverse components in the group velocity of the Alfvén wave, as well as to the appearance of a longitudinal component in the wave magnetic field.

2. For $|\tau| \ll 1$ the existence of the drift mirror mode is possible, as a spatially isolated structure in plasma. However, this quantity is a combination of the terms of order unity, which makes the inequality $|\tau| \ll 1$ appear to be rather artificial. On the other hand, the the magnetosheath plasma has a high $\beta$ value and low anisotropy (Narita and Glassmeier, 2005), so this condition is not so inconceivable.

3. The radial component of the wave vector is included in the Alfvén mode operator. But the plasma inhomogeneity leads to a "coupling" of these two modes, and therefore transverse dispersion of the drift mirror mode appears.

4. The Alfvén wave coupling provides the opportunity for inspecting the spatial structure of the drift mirror mode. In particular, plasma regions are considered in which the mode is trapped across magnetic shells, while being confined between two turning points. An equation is obtained describing the radial structure of the mode in such a resonator. The eigen value of this boundary problem gives the wave frequency value, including its imaginary part, the instability growth rate. The radial wavelength in such a resonator is determined by the plasma inhomogeneity scale $l$ and the azimuthal component of the wave vector $k_{y}, \lambda \sim \sqrt{l / k_{y}}$. In the general case, $\lambda$ has a real and an imaginary part. This means that the wave propagates across magnetic shells (with no energy transfer involved). The wave amplitude is modulated along the radial coordinate by the Gauss function.

5. The influence of the Alfvén mode leads to modification of the mirror instability criterion. In the "classical" theory, which does not include coupling, this instability develops if the condition $\tau<0$ is met. Coupling to the Alfvén mode for low harmonic numbers, $n \sim 1$, favours instability due to the finite value of $\Omega_{c}$. Note that anisotropy is often small in the 
Earth's magnetosphere and it is the $\Omega_{c}$ value that should be the major contributor to instability. The real part of the eigenfrequency grows slowly with increasing harmonic number. On the order of magnitude, $\omega$ coincides with the drift frequency $\omega_{*}$.

6. Drift mirror mode with $k_{r} \simeq 0$ can only exist if the functions $\omega_{*}(r)$ and $\gamma_{M}(r)+\Omega_{c}(r)$ have extrema with practically exactly coinciding locations. These extrema must also be in the region where $|\tau| \ll 1$. These coincidences are, of course, extremely unlikely. Moreover, another restriction to the plasma parameters arises from the inequality $\gamma \ll k_{\|} V_{\|}$: the value of $\Omega_{c}$ should be small compared to $k_{\|} V_{\|}$. But this is only possible if the rather rigid condition $k_{\|} l \gg 1$ is satisfied. This increases the doubts about whether some waves in space plasma are correctly interpreted as drift mirror modes.

Acknowledgements. The author is grateful to L. Chen, who initiated the author's research of the global structure of MHD waves in gyrokinetics and provided guidance during D. Klimushkin's stay in University of California at Irvine, to V. Mazur for his critical comments that allowed a number of errors to be avoided, and to P. Mager for discussion of the paper. The work was supported by the Russian Foundation for Basic Research under grant No 04-05-64321-a and Program of presidium of Russian Academy of Sciences \#16 and OFN RAS \#16.

Topical Editor I. A. Daglis thanks two referees for their help in evaluating this paper.

\section{References}

Antonsen, T. M and Lane, B.: Kinetic equations for low frequency instabilities in inhomogeneous plasma, Phys. Pluids, 23, 12051214, 1980.

Catto, P. J., Tang, W. M., and Baldwin, D. E.: Generalized gyrokinetics, Plasma Phys., 23, 639-650, 1981.

Chen, L. and Hasegawa, A.: Kinetic theory of geomagnetic pulsations, 1, Internal excitations by energetic particles, J. Geophys. Res., 96, 1503-1512, 1991.

Denton, R. E.: ULF waves in the magnetosheath, Int. J. Geomagn. Aeron., 2(1), doi:10.1029/GAI00340, 2000.

Ferrierè, K. M. and André, N.: A mixed magnetohydrodynamickinetic theory of low-frequency waves and instabilities in stratified, gyrotropic, two-component plasmas, J. Geophys. Res., 108(A7), 1308, doi:10.1029/2003JA009883, 2003.

Ferrierè, K. M. and André, N.: Low-frequency waves and instabilities in stratified, gyrotropic plasmas with arbitrary distribution functions, J. Geophys. Res., 110, A12201, doi:10.1029/2005JA011404, 2005.
Hasegawa, A.: Plasma instabilities and nonlinear effects, SpringerVerlag, Berlin-Heidelberg-New York, 1975.

Hasegawa, A.: Drift mirror instability in the magnetosphere, Phys. Pluids, 12, 2642-2650, 1969.

Klimushkin, D. Yu.: Spatial structure of small-scale azimuthal hydrodynamic waves in an axisymmetric magnetospheric plasma with finite pressure, Plasma Phys. Rep., 23, 858-871, 1997.

Leonovich, A. S. and Mazur, V. A.: A theory of transverse small scale standing Alfvén waves in an axially symmetric magnetosphere, Planet. Space Sci., 41, 697-717, 1993.

Lin, C. S. and Parks, G. K.: The coupling of Alfvén and compressional waves, J. Geophys. Res., 83, 2628-2636, 1978.

Lucek, E. A., Constantinescu, D., Goldstein, M. L., Pickett, J., Pincon, J. L., Sahraoui, F., Treumann, R. A., and Walker, S. N.: The Magnetosheath, Space Sci. Rev., 118, 95-152, 2005.

Mager, P. N. and Klimushkin, D. Yu.: Theory of azimuthally smallscale Alfvén waves in an axisymmetric magnetosphere with small but finite plasma pressure, J. Geophys. Res., 107, 1356, doi:1029/2001JA009137, 2002.

Narita, Y. and Glassmeier, K.-H.: Dispersion analysis of lowfrequency waves through the terrestrial bow shock, J. Geophys. Res., 110, A12215, doi:10.1029/2005JA011256, 2005.

Pokhotelov, O. A., Balikhin, M. A., Treumann, R. A., and Pavlenko, V. P.: Drift mirror instability revisited, 1, Cold electron temperature limit, J. Geophys. Res., 106, 8455-8463, 2001.

Pokhotelov O. A., Sagdeev, R. Z., Balikhin, M. A., and Treumann, R. A.: Mirror instability at finite ion-Larmor radius wavelengths, J. Geophys. Res., 109, A09213, doi:10.1029/2004JA010568, 2004.

Southwood, D. J. and Kivelson, M. G., Mirror instability: 1. Physical mechanism of linear instability, J. Geophys. Res., 98, 91819187, 1993.

Takahashi, K.: New observations, new theoretical results and controversies regarding Pc3-5 waves, Adv. Space Res., 17, (10)63(10)71, 1996.

Treumann, R. A.: Magnetic mirror modes: A superconducting analogue, in: Proc. of the Sixth Intern. School/Symposium on Space Plasma Simulation, edited by: Buchner, J., Dum, C. T., Scholer, M., Copernicus Gesellschaft, Katlenburg Lindau, 368371, 2001.

Treumann, R. A. and Baumjohann, W.: Collisionless mirror mode trapping, Nonlin. Proc. Geophys., 7, 179-184, 2000.

Treumann, R. A., Jaroschek, C. H., Constantinescu, O. D., Nakamura, R., Pokhotelov, O. A., and Georgescu E.: The strange physics of low frequency mirror mode turbulence in the high temperature plasma of the magnetosheath, Nonlin. Proc. Geophys., 11, 647-657, 2004.

Vetoulis, G. and Chen, L.: Kinetic theory of geomagnetic pulsations, 3, Global analysis of drift Alfvén-ballooning modes, J. Geophys. Res., 101, 15 441-15 456, 1996.

Woch, J., Kremser, G., and Korth, A.: A comprehensive investigation of compressional ULF waves observed in the ring current, $\mathbf{J}$ Geophys. Res., 95, 15 113-15 132, 1990. 